\title{
IMPACT OF NUCHAL CORD ON INTRAPARTUM COMPLICATIONS, MODE OF DELIVERY AND PERINATAL OUTCOME
}

\author{
Amita Gupta1, Tania Kakkar², Hema Pathania ${ }^{3}$, Virender Singh ${ }^{4}$ \\ ${ }_{1}^{1}$ Associate Professor, Department of Obstetrics and Gynaecology, SMGS Hospital, Government Medical College, Jammu. \\ ${ }^{2}$ Consultant Gynaecologist, Director Health Services, Jammu. \\ ${ }^{3}$ Medical Officer, Department of Obstetrics and Gynaecology, SMGS Hospital, Government Medical College, Jammu. \\ ${ }^{4}$ Consultant Paediatrics, Director Health Services, Jammu.
}

\begin{abstract}
BACKGROUND
Umbilical cord forms the connecting link between foetus and placenta through which blood flows to and from the placenta and is the lifeline of foetus. Cord accidents lead to $5-18 \%$ of all fatal foetal asphyxia cases and $10 \%$ of stillbirths, of which nuchal cord is one of the most common finding. A nuchal cord occurs when umbilical cord becomes wrapped 360 degrees around the foetal neck. The loops of cord may be - single or multiple; tight or loose. The present study was carried out to find out the impact of nuchal cord on intrapartum complications, mode of delivery and perinatal outcome.

Objectives- To assess the intrapartum complications, mode of delivery \& perinatal outcome in pregnancies with nuchal cord and to compare them in pregnancies without nuchal cord.
\end{abstract}

ABSTRACT

\section{MATERIALS AND METHODS}

The present study was prospective observational study conducted in the Department of Obstetrics and Gynaecology, SMGS Hospital, Govt. Medical College, Jammu. This study included all singletons, term pregnancies with cephalic presentation who delivered in emergency. It included 700 women out of which women with nuchal cord were taken as study group and women without nuchal cord were taken as control group. Outcome was assessed in terms of foetal heart rate irregularities, meconium staining of liquor, instrumental deliveries, caesarean section rates, weight of baby, Apgar scores at 1 and 5 minutes and NICU admissions. Outcome was also compared between subgroups i.e. single and multiple turns of cord; and tight and loose.

\section{RESULTS}

There was higher rate of foetal heart rate irregularities among study group as compared to control group. The rate of instrumental deliveries and caesarean section was more in study group as compared to control group. There was higher rate of meconium staining of liquor in multiple/tight subgroup as compared to single/ loose subgroup. NICU admissions were significantly higher in multiple/tight subgroup as compared to single/ loose subgroup.

\section{CONCLUSION}

Loose/Single nuchal cord may not be associated with adverse perinatal outcome. However, tight/multiple nuchal cord is associated with increased risk of adverse perinatal outcome.

\section{KEYWORDS}

Nuchal Cord, Perinatal Outcome.

HOW TO CITE THIS ARTICLE: Gupta A, Kakkar T, Pathania H, et al. Impact of nuchal cord on intrapartum complications, mode of delivery and perinatal outcome. J. Evolution Med. Dent. Sci. 2018;7(09):1114-1117, DOI: 10.14260/jemds/2018/253

\section{BACKGROUND}

Umbilical cord forms the connecting link between foetus and placenta through which blood flows to and from the placenta and is the lifeline of foetus.

Umbilical cord abnormalities are numerous. Cord accidents lead to $5-18 \%$ of all fatal foetal asphyxia cases and $10 \%$ of stillbirths, of which nuchal cord is one of the most common finding.(1,2) A nuchal cord occurs when umbilical cord becomes wrapped 360 degrees around the foetal neck. Nuchal cords are very common, with prevalence rates ranging between $6 \%$ to $37 \%$. $^{(3)}$

'Financial or Other Competing Interest': None.

Submission 21-12-2017, Peer Review 14-02-2018,

Acceptance 20-02-2018, Published 26-02-2018.

Corresponding Author:

Dr. Tania Kakkar,

House No. 239, Street No. 9,

Shakti Nagar,Jammu.

E-mail: virta6417@gmail.com

DOI: $10.14260 /$ jemds $/ 2018 / 253$

\section{Nuchal Cord is of Two Types-}

1. Type A- nuchal cord is wrapped around foetal neck 360 degrees.

2. Type B - it is described as hitch which can't be undone and ends up as a true knot.(4)

The loops of cord may be - single or multiple; tight or loose. Standard medical management recommends one attempt to slip the cord over the infant's head just before delivery of the body or, should the cord be too tight, to clamp and cut prior to delivery of the shoulders. (5)

If the umbilical cord becomes overly stretched or compressed during labour, it usually causes the baby's heart rate to slow down temporarily, which is not harmful. If the baby's heart rate slows to below 100 beats per minute and does not return to normal (120 to 160 for most babies) within a few minutes, measures like giving oxygen, fluid to the mother, changing her position, giving medications to slow down the contractions should be used. If there is still concern about pressure on the umbilical cord, a caesarean delivery may be needed. 
The ultrasound diagnosis of a cord around the neck was first described in 1982.(6) With the help of colour Doppler ultrasound, nuchal cord can be detected with a sensitivity of over $90 \%$. (7)

Several studies have been done to analyse deliveries with nuchal cord and have yielded varied results. Some authors reported that the nuchal cord is associated with increased risk of foetal distress, meconium staining of liquor, nonreassuring foetal heart rate patterns, slow progress of labour, Caesarean section rates, perinatal morbidity and mortality. $(8,1,9,2,10,11)$ while others did not find any significant increase in said complications. $(12,13,14,15,16)$

The insufficiency in data regarding the role of nuchal cord in foetal morbidity and mortality is a source of anxiety and frustration to both parturient and healthcare professionals. Considering the above facts, the present study was carried out to find out the impact of nuchal cord on intrapartum complications, mode of delivery and perinatal outcome.

\section{MATERIALS AND METHODS}

Design- Prospective observational study

Study Period- One year.

The present study was conducted in Department of Obstetrics and Gynaecology, SMGS Hospital, Govt. Medical College Jammu, during the period of October 2013 to September 2014. This study included all singletons, term pregnancies with cephalic presentation who deliver in the emergency once a week. The particulars of the patients were noted according to the prescribed proforma.

At the time of birth, cord around neck was noted. In babies with nuchal cord, the number of loops, and the type of cord that is tight or loose was also noted. The patients with nuchal cord at the time of delivery were taken as study group and the patients without nuchal cord were taken as control group. Outcome was assessed in terms of-

- FHR irregularities.

- Meconium staining of liquor.

- Instrumental delivery.

- Caesarean section rates.

- Weight of baby.

- Apgar score at 1 and 5 minutes.

- NICU admissions.

\section{Statistical Analysis}

The data was analysed using computer software SPSS 16.0. Results were expressed as percentage and mean \pm (standard deviation). All results were analysed statistically with the help of Chi-square test and student $t$ test. The difference was considered significant at $\mathrm{P}<0.05$.

\section{RESULTS}

A total of 700 patients were included in the study out of which 164 were having nuchal cord $(23.43 \%)$ and remaining $536(76.57 \%)$ were without nuchal cord. Out of 164 women in study group, single loop was present in 119 women (72.56\%), double loops in 38 women (23.17\%), and triple loops in 7 women (4.27\%). Loose loop was present in 99 women $(60.37 \%)$ and tight loop was present in 65 women (39.63\%). The difference between mean maternal age in the study group (25.61 \pm 0.26 years) and the control group (25.47 \pm 0.16 years) was insignificant $(p=0.667)$.
There were 65 (39.63\%) primigravidae in study group as compared to 237 (44.22\%) primigravidae in control group. The difference in gravidity between two groups was insignificant $(\mathrm{p}=0.299)$.

In study group, 88(53.66\%) newborns were male and $76(46.34 \%)$ were female. In control group 306(57.09\%) newborns were male as compared to $230(42.91 \%)$ females. The difference between two groups was statistically insignificant $(\mathrm{p}=0.472)$.

FHR Irregularities were present in 59 (35.98\%) women in the study group as compared to $86(16.04 \%)$ in the control group; $10(10.10 \%)$ women in NC loose subgroup as compared to $49(75.38 \%)$ women in NC tight subgroup; 28 $(23.53 \%)$ women in NC single sub group as compared to $31(68.89 \%)$ women in NC multiple sub group. The difference in FHR Irregularities between all the individual groups was statistically significant $(\mathrm{p}<0.0001)$.

Meconium staining of liquor was present in 21 (12.80\%) women in study group as compared to $65(12.13 \%)$ women in control group. The difference in meconium staining of liquor between two groups was statistically insignificant $(\mathrm{p}=0.817)$. However, it was present in $1(1.01 \%)$ women in NC loose subgroup as compared to $20(30.77 \%)$ in NC tight subgroup and in $5(4.20 \%)$ women in NC single subgroup as compared to $16(35.56 \%)$ women in NC multiple subgroup. The difference in meconium staining of liquor between these two sub groups was statistically significant $(\mathrm{p}<0.0001)$.

Vaginal delivery was the most common mode of delivery in both study and control group, its incidence was $60.37 \%$ in the study group and $71.83 \%$ in the control group. Caesarean section rate was $32.31 \%$ in the study group as compared to $25.75 \%$ in the control group. Rate of ventouse delivery was $4.88 \%$ in the study group as compared to $2.05 \%$ in the control group. Whereas rate of Forceps delivery was $2.44 \%$ in study group as compared to $0.37 \%$ in control group. The difference in mode of deliveries between two groups was statistically significant $(\mathrm{p}=0.006)$.

The rate of vaginal delivery in NC loose subgroup was $75.76 \%$ as compared to $36.92 \%$ in NC tight subgroup. Caesarean section rate was $23.23 \%$ in NC loose subgroup as compared to $46.15 \%$ in NC tight sub group. There was no ventouse delivery in $\mathrm{NC}$ loose subgroup as compared to $12.31 \%$ in NC tight subgroup. The rate of forceps delivery in NC loose subgroup was $1.01 \%$ as compared to $4.62 \%$ in NC tight subgroup. The difference in mode of deliveries between two groups was statistically significant $(\mathrm{p}<0.0001)$.

The rate of vaginal delivery in NC single subgroup was $72.27 \%$ as compared to $28.89 \%$ in NC multiple subgroup. Caesarean section rate was $26.89 \%$ in NC single subgroup as compared to $46.67 \%$ in NC multiple subgroup. Ventouse delivery rate was $0.84 \%$ in NC single sub group as compared to $15.56 \%$ in NC multiple subgroup. There was no forceps delivery in NC single subgroup as compared to $8.89 \%$ in NC multiple subgroup. The difference in mode of deliveries between two groups was statistically significant $(\mathrm{p}<0.0001)$. The difference in mean birth weight among all the groups was statistically insignificant.

The Apgar score $<7$ at 1 minute in study group was present in $6.10 \%$ newborns as compared to $3.17 \%$ in control group. The difference between two groups was statistically insignificant ( $\mathrm{p}=0.104)$. 
The Apgar score $<7$ at 5 minutes in study group was present in $1.22 \%$ of newborns as compared to $0.37 \%$ in control group. The difference between two groups was statistically insignificant $(\mathrm{p}=0.235)$. There was no newborn with Apgar score $<7$ at 1 minute in NC loose subgroup as compared to $15.38 \%$ in NC tight subgroup. The difference between two subgroups was statistically significant $(\mathrm{p}<0.0001)$. There was no newborn with Apgar score $<7$ at 5 minutes in NC loose subgroup as compared to $3.08 \%$ in NC tight subgroup. The difference between two subgroups was statistically insignificant $(\mathrm{p}=0.156)$.

There was no newborn with Apgar score $<7$ at 1 minute in NC single subgroup as compared to $22.22 \%$ in NC multiple subgroups. The difference between two subgroups was statistically significant $(\mathrm{p}=0.001)$. There was no newborn with Apgar score $<7$ at $5 \mathrm{~min}$ in NC single subgroup as compared to $4.44 \%$ in NC multiple sub group. The difference between two subgroups was statistically insignificant $(\mathrm{p}=0.114)$.

$7.32 \%$ of newborns in the study group needed NICU admission as compared to $4.85 \%$ in control group. The difference in incidence of NICU admission between two groups was statistically insignificant $(\mathrm{p}=0.238)$.

None of the newborns required NICU admission in NC loose sub group as compared to $18.46 \%$ in NC tight subgroup. The difference in incidence of NICU admission between single and tight subgroup was statistically significant $(\mathrm{P}<0.0001)$. Similarly, none of the newborns in NC single subgroup required NICU admission as compared to $26.67 \%$ in NC multiple subgroup. The difference in incidence of NICU admission between two subgroups was statistically significant $(\mathrm{p}<0.0001)$.

\section{DISCUSSION}

The prevalence of nuchal cord was $23.43 \%$ in the present study which is approximately similar to prevalence reported by Shrestha NS et al.(15) and Miser(12) i, e 22.85\% and 23.7\% respectively. Prevalence of nuchal cord reported by Bernard ES et al.(17) was $8 \%$, and was $48.28 \%$ by Rauf B et al.(18) In the present study, number of loops varied from 1 to 3 . The prevalence of single loop of cord around neck was $72.56 \%$ and of multiple loops was $27.44 \%$. Shrestha NS et al.(15) reported prevalence of single loop and of multiple loops as $82.90 \%$ and $17.10 \%$ respectively. In another study done by Rauf B et al. (2013),(18) Prevalence of single loop was $87.5 \%$ and of multiple loops was $12.5 \%$.

Of 164 cases, loose nuchal cord was present in $60.37 \%$ cases and tight nuchal cord was present in the rest. Whereas Shrestha NS et al.(15) reported prevalence of loose loop and tight loop as $76.07 \%$ and $23.93 \%$ respectively. Prevalence of loose loop and tight loop was reported as $89.3 \%$ and $10.7 \%$ by Rauf B et a.(18)

In the present study, FHR Irregularities were present in $35.98 \%$ women in study group as compared to $16.04 \%$ in control group. The difference in FHR irregularities between two groups is statistically significant $(p<0.0001)$. These findings were similar to findings reported by Sheiner et al.(14) $(p<0.001)$ and Rauf B et al.(18) $(p=0.009)$. The incidence of FHR Irregularities in multiple/tight subgroup as compared to single/loose subgroup was higher and statistically significant $(\mathrm{p}<0.0001)$ in our study. These were similar to findings reported by Kashyap M et al.(11) and Singh G et al.(10)
Meconium staining of liquor was present in $12.80 \%$ women in study group as compared to $12.13 \%$ women in control group which was similar to findings reported by Singh G et al. (2008).(10). The incidence of meconium staining of liquor in multiple/tight subgroup as compared to single/loose subgroup was higher and statistically significant $(p<0.0001)$ in the present study. These were similar to findings reported by Kashyap M et al.(11)

The most common mode of delivery was vaginal in both study and control groups. There was higher rate of instrumental and caesarean deliveries in multiple/tight subgroup as compared to single/loose subgroup ( $\mathrm{p}<0.0001)$. These findings are similar to the study done by Kashyap M et al.(11) which reported vaginal delivery in $75 \%$ cases, caesarean in $24 \%$ and forceps delivery in $1 \%$ of study group. In control group $91 \%$ had vaginal delivery, caesarean in rest 9\%. Larson JD et al.(19) also reported higher incidence of operative deliveries in multiple nuchal cord $(p<0.001)$. Higher incidence of instrumental deliveries in study group as compared to control group were also reported by Rauf B et al. (18) and Singh G et al.(10) $(\mathrm{p}=0.01)$. On the contrary, Begum AA et al.(20) and Shrestha NS et al.(15) did not find any significant association between nuchal cord and mode of delivery.

In the present study there was higher incidence of $\mathrm{A} / \mathrm{S}<7$ at 1 minute for multiple/tight NC subgroup as compared to single/loose NC subgroup and the difference was also statistically significant $(\mathrm{p}<0.0001)$. Singh $\mathrm{G}$ et al.(10) found significant correlation between loose and tight subgroup for $\mathrm{A} / \mathrm{S}<7$ at 1 minute $(\mathrm{p}<0.001)$.

Interestingly, $\mathrm{A} / \mathrm{S}<7$ at $5 \mathrm{~min}$ did not show any significant difference between tight and loose subgroup, in the present study. Singh G et al.(10) $(\mathrm{p}=0.05)$ and Singh G et al.(21) $(\mathrm{P}<0.1)$ also found the similar correlation. So, it was taken into account that the effect of nuchal cord on Apgar scoring was transient.

In the present study it was observed that, there was no statistically significant difference in NICU admissions between the study group and the control group ( $p=0.238$ ).

None of the newborns in single/loose NC sub group required NICU admission as compared to $26.67 \%$ in the multiple subgroup and $18.46 \%$ in tight subgroup, who required NICU care. The difference in NICU admissions between two subgroups was statistically significant $(\mathrm{p}<0.0001)$. In a study done by Henry E et al. term neonates with a tight nuchal cord were slightly more likely to be admitted to a Neonatal Intensive Care Unit (6.6\% vs 5.9\% admission rate, $\mathrm{P}=0.0001)$. So it was perceived that tight/multiple nuchal cord might affect NICU admission rate because of higher incidence of meconium and low Apgar scores.

\section{CONCLUSION}

We conclude that the nuchal cords occur quite commonly. Loose/Single nuchal cord may not be associated with adverse perinatal outcome. However, tight/multiple nuchal cord is associated with increased risk of low Apgar score at 1 minute and increased incidence of foetal distress with an associated increased transfer rates to neonatology unit. There was no significant evidence to incriminate the cord around neck to be responsible for the perinatal death. However, such patients require close monitoring during labour, preferably by continuous foetal electronic heart rate monitoring as well as 
strict maintenance of partogram. The presence of a nuchal cord per se is not found to be an indication of operative delivery but as tight and multiple nuchal loops were seen to be associated with higher incidence of foetal distress, low Apgar scores and increased rate of NICU admission, caesarean section can be considered in such patients.

\section{REFERENCES}

[1] Clapp JF, Stepanchak W, Hashimoto K, et al. The natural history of antenatal nuchal cords. Am J Obstet Gynecol 2003;189(2):488-93.

[2] Onderoğlu LS, Dursun P, Durukan T. Perinatal features and umbilical cord blood gases in newborns complicated with nuchal cord. Turk J Pediatr 2008;50(5):466-70.

[3] Peregrine E, O'Brien P, Jauniaux E. Ultrasound detection of nuchal cord prior to labor induction and the risk of caesarean section. Ultrasound Obstet Gynecol 2005;25(2):160-4.

[4] Collin JH. Umbilical cord accidents: human studies. Semin Perinatol 2002;26(1):79-82.

[5] Schorn M, Blanco J. Management of the nuchal cord. J Nurse Midwifery 1991;36(2):131-2.

[6] Jouppila P, Kirkinen P. Ultrasonic diagnosis of nuchal encirclement by the umbilical cord: a case and methodological report. J Clin Ultrasound 1982;10(2):59-62.

[7] Aksoy U. Prenatal color Doppler sonographic evaluation of nuchal encirclement by the umbilical cord. J Clin Ultrasound 2003;31(9):473-7.

[8] Shepherd AJ, Richardson CJ, Brown JP. Nuchal cord as a cause of neonatal anemia. Am J Dis Child 1985;139(1):71-3.

[9] Ogueh O, Al-Tarkait A, Vallerand D, et al. Obstetrical factors related to nuchal cord. Acta Obstet Gynecol Scand 2006;85(7):810-4.
[10] Singh G, Dasgupta E. Nuchal cord and its outcome: a retrospective analysis. J Obstet Gynecol India 2008;58(3):244-7.

[11] Kashyap M, Meena P. Fetomaternal outcome in primigravidae having single loop of cord around neck. IJOSR 2013;1(6):4-14.

[12] Miser WF. Outcome of infants born with nuchal cords. J Fam Pract 1992;34(4):441-5.

[13] Gonzalez-Quintero VH, Tolaymat L, Muller AC, et al. Outcomes of pregnancies with sonographically detected nuchal cords remote from delivery. J Ultrasound Med 2004;23(1):43-7.

[14] Sheiner E, Abramowicz JS, Levy A, et al. Nuchal cord is not associated with adverse perinatal outcome. Arch Gynecol Obstet 2006;274(2):81-3.

[15] Shrestha NS, Singh N. Nuchal cord and Perinatal outcome. Kathmandu Univ Med J (KUMJ) 2007;5(3):360-3.

[16] Zahoor F, Minhas Z, Zaki A. Perinatal outcome of nuchal cord. J Postgrad Med Inst 2013;27(2):174-8.

[17] Bernad ES, Craina M, Tudor A, et al. Perinatal outcome associated with nuchal umblical cord. Clin Exp Obstet Gynecol 2012;39(4):494-7.

[18] Rauf B, Akhter R. Frequency of Nuchal cord at delivery between 36 to 41 weeks and to assess perinatal outcome. KJMS 2013;1:28-31.

[19] Larson JD, Rayburn WF, Crosby S, et al. Multiple nuchal cord entanglements and intrapartum complications. Am J Obstet Gynecol 1995;173(4):1228-31.

[20] Begum AA, Sultana H, Hasan R, et al. Clinical study of fetal outcome in cases of nuchal cord. JAFMC Bangladesh 2011;7(1):25-7.

[21] Singh G, Sidhu M. Nuchal cord: a retrospective analysis. MJAFI 2008;64(3):237-40. 